\title{
Um Sistema de Recomendação Híbrido Integrado a Ontologia em um Ambiente de Aprendizagem Ubíqua
}

\author{
Sidney D.F. Bezerra, Antônio V. T. Costa, F. Milton M. Neto, \\ Patrício A. Silva, Bruno de S. Monteiro \\ Departamento de Ciências Exatas e Naturais - Universidade Federal Rural do Semi- \\ Árido (UFERSA) Caixa Postal 59.625-900 - Mossoró - RN - Brasil \\ \{sidney.firmino, antonio.costa, miltonmendes, patricio.alencar, \\ brunomonteiro\} @ufersa.edu.br
}

\begin{abstract}
Recommendation systems (SR) has the purpose of analyzing a set of items and check which of these are the most relevant to a consumer. For this work was developed a Hybrid SR integrated into an ontology that manages the learner profile information and the contents of the YouTube and Wikipedia databases. For validation, the proposed SR was implemented as a webservice and integrated to the architecture of a ubiquitous learning environment (Edubi), which allowed to be tested by students of a higher education institution through the Edubi-Web and Edubi-Mobile applications. The results obtained after qualitative collection show indications of the feasibility of the proposal.
\end{abstract}

Resumo. Os Sistemas de Recomendação (SR) tem o propósito de analisar um conjunto de itens e verificar quais destes são os mais relevantes para um consumidor. Para este trabalho foi desenvolvido um SR Hibrido integrado a uma ontologia que gerencia as informações de perfil do aprendiz e de conteúdos provenientes do Youtube e da Wikipédia. Para validação, o SR proposto foi implementado como um webservice e integrado à arquitetura de um ambiente de aprendizagem ubíqua (Edubi) o que permitiu ser testado por estudantes de uma instituição de ensino superior, por meio das aplicações Edubi-Web e Edubi-Mobile. Os resultados obtidos, após coleta e análise de dados qualitativa, apresentam indícios de viabilidade da proposta.

\section{Introdução}

Com a popularização de Tecnologias de Informação e Comunicação (TIC), é impossível ignorar sua influência e potencial no contexto educacional, tanto na modalidade presencial, quanto, e principalmente, na modalidade a distância. (GLUZ, 2017). Neste sentido, a aprendizagem ubíqua (ubiquity learning ou u-learning) surge da combinação de distintos fundamentos tecnológicos com o objetivo de prover ferramentas que ampliam as oportunidades de práticas de ensino e aprendizagem. Ferramentas baseadas nesse conceito permitem oferecer aos aprendizes e professores conteúdos e serviços relevantes com base no contexto no qual os sujeitos estão inseridos (MONTEIRO, 2015). 
VII Congresso Brasileiro de Informática na Educação (CBIE 2018)

Anais do XXIX Simpósio Brasileiro de Informática na Educação (SBIE 2018)

Devido ao crescimento da produção de dados na web, tornou-se essencial desenvolver procedimentos computacionais capazes de manipular, processar, gerenciar e armazenar informações com o propósito de relacioná-las com as características e interesses dos usuários. Entres esses procedimentos, encontram-se as ontologias e os Sistemas de Recomendação (SR). O processo de recomendar um item para um consumidor depende da coleta de dados, dos critérios de manipulação dos dados que foram usados para entregar os itens e de como é feito o processo de avaliação dos conteúdos (BARROS, 2016). O propósito de um SR é permitir que o consumidor receba, não somente um item buscado por uma consulta, mas também que o sistema seja capaz de prever um item que seja relevante aos seus interesses (GARIN, 2006).

A utilização de ontologias em SR proporciona a ampliação e a representação semântica dos usuários e conteúdos. Uma ontologia tem como função descrever as informações de um domínio específico, possibilitando que o seu conceito seja utilizado distintamente por pessoas, bases de dados e aplicações de um domínio. Assim, quando aplicações necessitam das informações da ontologia, pode-se fazer reutilização das suas representações, em vez de ter que definir tudo novamente (REZENDE, 2015).

Deste modo, este trabalho apresenta um Sistema de Recomendação Híbrida de Conteúdos Hipermídia Baseado em Ontologia (SRHCHBO). O sistema de recomendação proposto é capaz de analisar as características dos usuários (estudantes, tutores e professores) e os metadados dos conteúdos Youtube e Wikipédia, de modo a selecionar conteúdos por técnicas de filtragem. Para validação, o SR proposto foi integrado ao Edubi (EDUBI, 2018), por ser um ambiente de aprendizagem ubíqua composto por quatro aplicações clientes (Web, Mobile, TV e Watch) e consome os serviços da Youubi API (MONTEIRO, 2015). Esta integração possibilitou uma melhor adaptação dos estudantes em ambientes educacionais, permitindo a personalização dos conteúdos de acordo com o contexto que o estudante está inserido. O uso de ontologia foi escolhido devido a sua flexibilidade, capacidade de modelar um conceito, reuso e de ser capaz de inferir um conceito a partir de um conjunto de informações.

O corrente trabalho está organizado da seguinte forma: A Seção 2 apresenta a ontologia utilizada par manipular as informações dos estudantes e dos conteúdos manipulados, demonstrando os elementos utilizados na criação desta. A Seção 3 apresenta a Youubi API e as aplicações Edubi que consomem essa API. A Seção 4 detalha o sistema de recomendação desenvolvido. A Seção 5 apresenta os resultados obtidos na pesquisa por intermédio de um grupo focal. Por fim, a Seção 6 traz as considerações finais acerca do desenvolvimento e contribuições do projeto.

\section{Ontologia PUYW: Perfil de Usuário, YouTube e Wikipédia}

O objetivo, primeiramente, foi representar o perfil do usuário, que pode ser um tutor, um professor, mas como persona principal, um estudante. Para isso, foram analisados atributos, informações, relacionamentos, localização, preferências, dentre outras. O modelo ontológico foi utilizado como forma de representar as informações de perfil e dos conteúdos manipulados em um ambiente de aprendizagem.

O desenvolvimento da ontologia se deu a partir da metodologia Ontology Development 101. Este procedimento consiste de um roteiro de desenvolvimento iterativo de fácil execução no processo de desenvolvimento de ontologias, dividido em 
VII Congresso Brasileiro de Informática na Educação (CBIE 2018)

Anais do XXIX Simpósio Brasileiro de Informática na Educação (SBIE 2018)

sete etapas (ISOTANI, 2015). O domínio da ontologia deste trabalho é voltado para um sistema de recomendação para armazenar as informações do aprendiz e os conteúdos acessados por eles. O escopo é apoiar o aprendizado de estudantes, armazenando e fornecendo informações acerca dos conteúdos de acordo com suas características, preferências e histórico de interações. Este trabalho fez o reuso de classes, propriedades e atributos da ontologia PERSONNA (REZENDE, 2015) em relação ao perfil do aprendiz.

A ontologia desenvolvida contém dez classes, são elas: Acessibilitiy (relacionada com as informações de acessibilidade dos aprendizes), Device (armazena os dados a respeito dos dispositivos utilizados pelos estudantes), Interest (relacionada com os interesses do aprendiz em determinados conteúdos), LearnerPersonalInformation (relacionada com os dados pessoais do aprendiz), Location (referente às informações da localização geográfica do aprendiz), Preferences (compreende as informações das preferências do aprendiz em relação às mídias do conteúdo), Social_relations (representa os relacionamentos entre aprendizes), Time (relacionada com as datas e o momento da realização de um evento do aprendiz), YoutubeContent (relacionada aos metadados disponibilizados pela API do Youtube (YOUTUBE-DEV, 2018) ) e WikipediaContent (relacionada com os metadados disponibilizados pela API do Mediawiki (MEDIAWIKI, 2018) ). Com o propósito de relacionar entre si essas classes, foram estabelecidas vinte e uma Object Properties e cinquenta e seis Data Properties. As propriedades Object Properties têm como função relacionar as classes da ontologia, permitindo que indivíduos de uma classe possam relacionar-se com outros indivíduos de classes diferentes.

Assim sendo, por meio desta representação ontológica, é possível gerenciar os usuários da aplicação, quais conteúdos eles acessaram, por quais canais de informações (Youtube ou Wikipédia) estes conteúdos foram transmitidos, que dispositivo foi utilizados, por qual tipo de recomendação foi recomendado um item, dentre outras representações.

\section{Ambiente de aprendizagem Ubíqua Edubi}

O ambiente Edubi (EDUBI, 2018), representado na Figura 1, é composto por quatro aplicações clientes (Web, Mobile, Watch e TV), cada uma delas com suas particularidades, e dois componentes de integração que possibilitam a essas aplicações consumirem os serviços disponibilizados por Moodle, Wikipedia e Youtube. Além desses, os artefatos de software do ambiente Edubi consomem também os serviços da Youubi API (MONTEIRO, 2015), componente central da arquitetura. Dentro desse contexto, o presente trabalho se concentrou especificamente na concepção e desenvolvimento do componente responsável por recomendar conteúdos do Youtube e da Wikipedia. 
VII Congresso Brasileiro de Informática na Educação (CBIE 2018)

Anais do XXIX Simpósio Brasileiro de Informática na Educação (SBIE 2018)

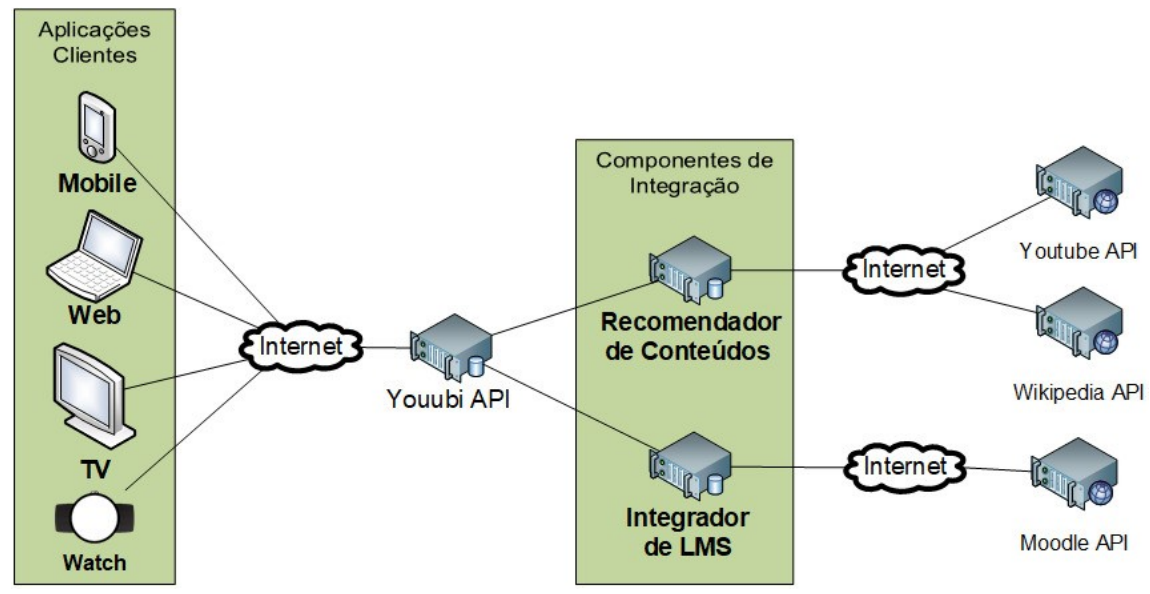

Figura 1. Visão geral do ambiente Edubi (EDUBI, 2018)

O processo de design do Edubi priorizou cenários de educação a distância, porém, pode ser adotado também na modalidade presencial, dentro do paradigma da aprendizagem ubíqua e informal. Para isso, faz uso de funcionalidades cujos serviços base são providos pela Youubi API.

O Youubi (MONTEIRO, 2015) é uma API (Application Programming Interface) que fornece serviços que auxiliam o desenvolvimento de aplicações clientes voltadas à computação ubíqua, mas que pode ser adotada em diversos domínios específicos (educação, saúde, entretenimento, turismo, comunicação, marketing, etc). Ela permite a criação e manipulação de sete Entidades Elementares (EE): pessoa, postagem, evento, pergunta, local, grupo e missão. Atualmente estão disponíveis 154 serviços (78 requisições post e 76 requisições get), que possibilitam às aplicações clientes implementarem as seguintes funcionalidades: autoria de conteúdo (postagens, eventos, perguntas e locais); recomendação das EE; rede social; comentários; chat; grupo de discussão (por meio da entidade grupo); notificações; gamificação (pontos de experiência, medalhas e rankings); missões (sequência de objetivos); geolocalização; bookmark (lista de conteúdos salvos); monitoramento quantitativo de interações; e moderação.

\subsection{Integração Edubi com o componente de Recomendação Híbrida}

Para garantir as propriedades de alta coesão e baixo acoplamento, os serviços do sistema de recomendação (SR) proposto são disponibilizados por meio de um webservice, conforme representado pelo "Recomendador de Conteúdos" da Figura 1. Desta forma, o SR pode consumir os serviços da Youubi API, como também o Youubi pode consumir os serviços oferecidos pela API do SR.

Para ilustrar essa integração, será descrito o cenário de criação de um novo conteúdo ou o cadastro de um novo usuário. Sempre que um novo usuário ou novo conteúdo é criado por meio de alguma aplicação cliente do Edubi, o Youubi consome um método do SR para informa-lo sobre os dados das entidades criadas. Em seguida, com base nesses dados, o SR atualiza a ontologia e executa seus algoritmos de recomendação (colaborativa e baseada em conteúdo); neste processo, o SR também pode consumir a Youubi API em busca de mais informações sobre a entidade recém criada ou sobre outros dados providos pela API. Em seguida, o SR consome os serviços 
VII Congresso Brasileiro de Informática na Educação (CBIE 2018)

Anais do XXIX Simpósio Brasileiro de Informática na Educação (SBIE 2018)

providos pela Wikipedia e Youtube em busca de conteúdos que combinem com o status atual da ontologia. Por fim, as referências dos conteúdos selecionados pelo SR são enviadas para o Youubi API para que fiquem disponíveis para os usuários, por meio das aplicações clientes. Entretanto, é importante salientar que os conteúdos selecionados pelo SR precisam ser validados primeiramente por um usuário moderador (professor ou tutor) para que possam, finalmente, ficarem visíveis para os demais usuários. A Figura 2 apresenta o painel do cliente Edubi-Web onde os moderadores podem aceitar ou não um conteúdo selecionado pelo SR.

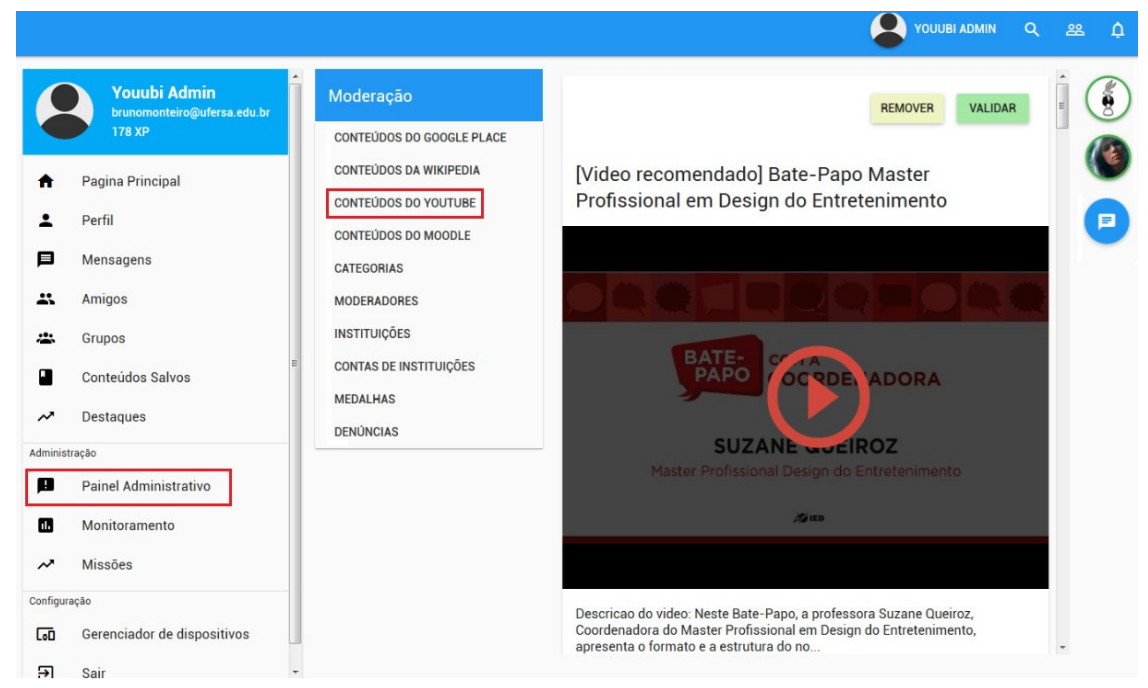

Figura 2. Tela de Moderação dos conteúdos recomendados automaticamente.

Além de ser provocado pela criação de novos conteúdos, o SR também seleciona novos conteúdos diante das seguintes interações do usuário (por meio das aplicações clientes do Edubi): "curtir" um conteúdo, "favoritar" um conteúdo, e aceitar outro usuário como amigo. Entretanto, interações negativas, tais como, denunciar um conteúdo, "descurtir" um conteúdo, "desfavoritar" um conteúdo e desfazer uma amizade, são informadas também ao SR, porém, apenas atualizam o status da ontologia, sem selecionar novos conteúdos.

Além desses cenários, outro fluxo de interação precisa ser realizado para evitar o cold-start. Este é um conhecido problema dos sistemas de recomendação que ocorre quando os dados dos usuários e dos itens são escassos ou não possuem uma classificação para se recomendar algo (BARJASTEH, 2015). Para evita-lo, o SR consome os serviços da Youubi API em busca das categorias que classificam os conteúdos. Assim, novos conteúdos (Youtube e Wikipédia) são selecionados e recomendados com base nesses dados mais genéricos.

\section{Sistema de Recomendação Híbrida de Conteúdos Hipermídia Baseado em Ontologia}

Com a finalidade de disponibilizar um mecanismo de recomendação de conteúdos, que pudesse ser usado por um sistema de baseado nos princípios da aprendizagem ubíqua, e que fizesse uso dos serviços e informações disponibilizados pelo Youtube e Wikipédia, foi concebido e desenvolvido um SR híbrido, baseado em ontologia. Para isso, sua 
VII Congresso Brasileiro de Informática na Educação (CBIE 2018)

Anais do XXIX Simpósio Brasileiro de Informática na Educação (SBIE 2018)

arquitetura (Figura 3) é composta por módulos de recomendação, em que cada módulo é responsável por uma técnica de filtragem de conteúdo e seleção. Fundamentalmente $\mathrm{o}$ SR proposto tem como função gerenciar as características dos usuários com o intuito de processar tais informações e, assim, recomendar conteúdos de acordo com o perfil. Para isso, ele gerencia as informações da ontologia PUYW, cujos atributos, apresentados na Seção 2, são constantemente atualizados.

O processo de recomendação é iniciado pelo acesso às informações externas do usuário, que podem ser acessadas por consultas à Youubi API. Essas informações são inseridas na ontologia (PUYW), com o objetivo de gerenciar os dados dos usuários. Quando o SR atualiza a ontologia PUYW, é iniciado o processo de análise das informações, que dá início ao processo de recomendação por meio de técnicas de filtragem e algoritmos de semelhanças entre usuários.

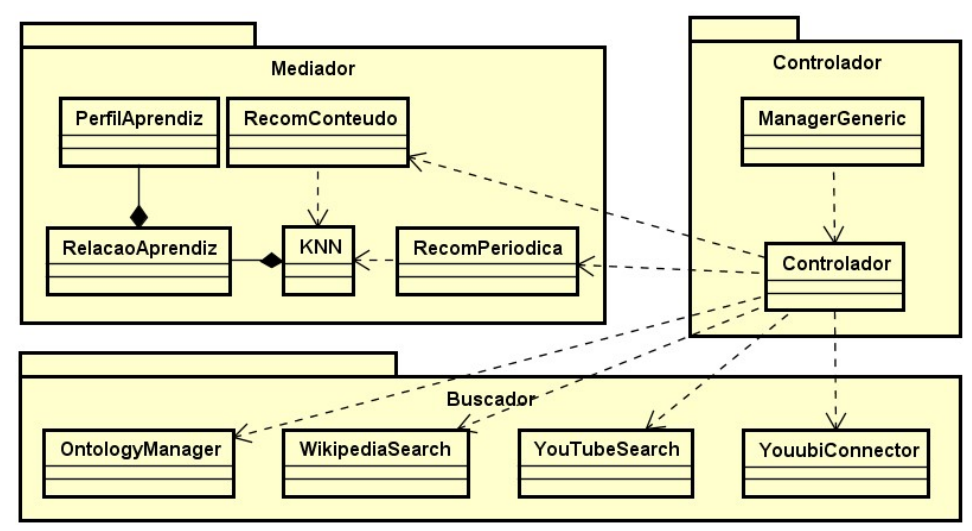

Figura 3. Arquitetura do componente de recomendação.

Sistemas de recomendação baseados em conteúdo representam a similaridade entre objetos que o usuário manuseou anteriormente (VIEIRA, 2012). A recomendação baseada em conteúdo realizada neste trabalho analisa os metadados de um usuário alvo e seleciona conteúdos nas bases do YouTube e Wikipédia que estejam de acordo com as características e o histórico de acesso. Esse processo é executado e produz uma lista de conteúdos recomendados.

As recomendações colaborativas realizam suas recomendações por meio de comparações entre os usuários e suas similaridades, isto é, os artefatos recomendados são aqueles que já foram utilizados anteriormente por usuários com perfis semelhantes (VIEIRA, 2012). A recomendação baseada em colaboração desenvolvida nesta pesquisa verifica os metadados do repositório semântico, bem como semelhanças entre usuários, conteúdos acessados e avaliados, os usuários mais semelhantes, entre outros. Esse procedimento produz uma lista de conteúdos avaliados com base na similaridade entre usuários em relação a um indivíduo alvo.

A recomendação híbrida combina os pontos fortes de dois ou mais métodos de recomendação, oferecendo melhores itens para o usuário e atendendo melhor suas necessidades (TARUS, 2017). A recomendação híbrida desenvolvida neste trabalho consiste em utilizar procedimentos distintos de filtragem de conteúdos, beneficiando-se de suas melhores práticas, no caso, são adotadas as abordagens híbridas: Pesada e Mista (BURKE, 2002). 
VII Congresso Brasileiro de Informática na Educação (CBIE 2018)

Anais do XXIX Simpósio Brasileiro de Informática na Educação (SBIE 2018)

A recomendação Pesada combina técnicas com o objetivo de produzir uma única recomendação. A recomendação Mista tem por objetivo recomendar conteúdos de forma simultânea por técnicas distintas. No SR proposto, são recomendadas três listas de conteúdos, de forma simultânea representando cada tipo de recomendação (Baseada em Colaboração, Baseada em Conteúdo e Híbrida Pesada e Mista), gerando vários conteúdos por diferentes procedimentos.

As ações feitas pelos usuários no Edubi modificam os conteúdos recomendados e a quantidade de conteúdos oferecidos. As avaliações positivas realizadas em conteúdos recomendados modificam a quantidade de itens oferecidos na próxima recomendação, adicionando ou removendo conteúdos em relação ao tipo de recomendação oferecida. Na primeira recomendação, são oferecidos conteúdos de forma igualitária para cada tipo. Após a primeira recomendação, as ações feitas pelos usuários nestes conteúdos irão influenciar nos pesos para os seguintes itens a serem recomendados. O cálculo é realizado levando em consideração o tipo de recomendação dos conteúdos, a partir das visualizações e avaliações feitas no conteúdo recomendado.

\section{Resultados}

Com o objetivo de avaliar os conteúdos recomendados por intermédio do Edubi, estudantes de uma instituição de ensino superior, do curso de ciência e tecnologia da Universidade Federal Rural do Semi-Árido (UFERSA), realizaram atividades, com acompanhamento do professor, fazendo uso das aplicações Edubi-Web e Edubi-Mobile, entre os dias 26 de março e 13 de abril de 2018. Ao final desse período, foi realizada apenas uma sessão de Grupo Focal, com 12 alunos participantes, estes interagiram entre si juntamente com o entrevistador. Nessa sessão, os alunos foram questionados sobre três tópicos relacionados aos conteúdos gerados pelo componente de recomendação, e disponibilizados para visualização e interação pelas aplicações Edubi.

O primeiro tópico se refere aos conteúdos recomendados pelo sistema, conforme Quadro 1. Foi avaliado se os conteúdos estavam de acordo com as necessidades dos estudantes, se foram suficientes e se facilitaram o aprendizado.

\section{Quadro 1. Avaliação dos estudantes sobre a aplicabilidade do Sistema Edubi com relação aos conteúdos do YouTube e Wikipédia recomendados.}

\section{RESPOSTAS}

"Os conteúdos recomendados estavam de acordo com a realidade da gente, dentro do período dos cursos ofertados como cálculo e geometria analítica."

"O sistema ficou bom em relação ao Youtube, mas ficou a desejar em relação aos artigos, somente foram recomendados vídeos."

"Estamos estudando Geometria e eu compartilhei e interagi com algumas postagens sobre esse conteúdo. Aí, o sistema foi além e colocou conteúdos dos próximos assuntos que iríamos estudar nas disciplinas, postagens que completavam os assuntos que estávamos estudando."

"A partir do que era postado, vídeo de cálculo ou geometria analítica, o sistema acrescentava novos vídeos relacionados sobre os conteúdos que eu estava estudando naquele momento."

"Em quantidade sim foram suficientes os conteúdos recomendados, no entanto mais vídeos, 
VII Congresso Brasileiro de Informática na Educação (CBIE 2018)

Anais do XXIX Simpósio Brasileiro de Informática na Educação (SBIE 2018)

uma deficiência em artigos."

"Além do que nós postávamos, eram apresentados novos conteúdos parecidos."

"Em relação ao conteúdo foi muito bom, estava muito pertinente com o que estávamos estudando, mas do Wikipédia não, não vi a recomendação escrita, mas vídeo. E Sim os conteúdos estavam de acordo com o meu curso."

De acordo com os resultados do primeiro tópico, verifica-se que os conteúdos recomendados por intermédio das aplicações Edubi estavam de acordo com as necessidades dos estudantes e coerentes com as disciplinas do seu curso, como também de seus interesses. Nos relatos, percebe-se que os conteúdos recomendados foram suficientes e ofertavam outros conteúdos semelhantes aos que estavam interessados. Contudo, foi observado que os artigos do Wikipédia não responderam às necessidades dos estudantes e que se deve melhorar as estratégias de recomendação desses itens.

O segundo tópico se refere à precisão das recomendações do sistema, conforme Quadro 2. Foi avaliado se o sistema apresentava algum erro nos conteúdos recomendados e se era capaz de identificar as mudanças de interesses dos estudantes.

Quadro 2. Avaliação dos estudantes sobre a aplicabilidade do Sistema Edubi com relação à precisão das recomendações do sistema.

\section{RESPOSTAS}

"Do Youtube o sistema foi preciso, no entanto, a Wikipédia teve poucos conteúdos, logo não achei preciso."

"Um dos erros que identifiquei foi em relação às novas postagens, ficavam no final das postagens do perfil, eu tinha que baixar tudo para ver a nova postagem, eu tinha que visualizar as outras postagens tudo de novo."

"O sistema não apresentou erro nas recomendações, pelo menos não vi algo fora do comum."

"O sistema não atualiza automaticamente, tinha que apertar para atualizar, para as postagens serem recebidas."

"Não deu para perceber se ocorreu mudança no sistema, se ele se adaptou ou não. Acho que foi pouco tempo de uso para que o sistema se adaptasse à mudança do meu interesse."

"Eu notei que alguém postou sobre empreendedorismo, e depois que eu interagir com o vídeo, sugiram novas postagens para mim então, percebi as adaptações no sistema nesse momento."

De acordo com os resultados do segundo tópico, é verificado que o sistema não apresentou erro em relação às recomendações dos conteúdos. No entanto, foi relatado que os novos conteúdos gerados nas aplicações eram apresentados no final do perfil, o que se torna uma atividade incômoda para o usuário devido ter que visualizar novamente as postagens antigas. Outra ocorrência relatada pelos estudantes foi no quesito de atualização automática dos conteúdos postados, em que o estudante teria que selecionar o botão de atualizar para que as novas postagens fossem recebidas.

Sobre as adaptações das aplicações, foi relatado que as interações feitas pelos estudantes no sistema modificaram os conteúdos que foram recomendados. O sistema conseguiu identificar a mudança de interesse dos estudantes, desse modo, consegue recomendar itens semelhantes aos que eles interagiram. Outro relato dos estudantes é 
VII Congresso Brasileiro de Informática na Educação (CBIE 2018)

Anais do XXIX Simpósio Brasileiro de Informática na Educação (SBIE 2018)

que o sistema é preciso em relação a recomendações de itens, que conteúdos gerados pelas aplicações foram capazes de identificar a colaboração das avaliações deles nas postagens, como também em identificar os conteúdos semelhantes que tinham interação e recomendar mais itens do interesse deles.

O terceiro e último tópico se refere à pontualidade das recomendações dos conteúdos feitos pelo sistema, conforme Quadro 3. Foi avaliado se o tempo de resposta do carregamento dos conteúdos ocorreu foi viável, se os conteúdos eram atualizados corretamente e se eram adequados ao ambiente de acesso (internet, localização e dispositivo).

\section{Quadro 1. Avaliação dos estudantes sobre a aplicabilidade do Sistema Edubi com relação à pontualidade do sistema.}

\section{RESPOSTAS}

"Eu não senti demora ao receber conteúdos, o tempo de resposta foi curto, quando olhava novamente já tinha novas sugestões de conteúdos."

"Foi bem rápido o sistema, eu curti os vídeos de umas postagens e depois foram recomendados novos conteúdos, após atualizar a página inicial."

"Tivemos conteúdos no tempo correto."

"As vídeos aulas era bem recentes, os vídeos não eram antigos."

"Preferia assistir no celular do que no computador as postagens de vídeo."

"Não teve diferenças no computador e celular sobre os conteúdos, os vídeos que foram recomendados eram curtos e bons para assistir."

"No celular e no computador, ambos estavam adaptadas para visualizar o conteúdo."

Conforme os resultados do terceiro tópico, pode-se verificar que os estudantes não relataram demora nas recomendações de novos itens e que estes eram atuais. Ainda acrescentaram que por meio das aplicações no computador e no smartphones era possível visualizar os conteúdos recomendados sem dificuldades.

\section{Conclusão}

Neste trabalho, foi apresentado um sistema de recomendação híbrida baseado em uma ontologia denominada PUYW. Este sistema foi encapsulado em um webservice e integrado a um ambiente de aprendizagem ubíqua, o Edubi, cujas aplicações puderam ser utilizadas por estudantes de uma instituição de ensino, no período de três semanas.

Como forma de avaliação do sistema desenvolvido, foi adotada a técnica qualitativa de grupo focal, fundamental para compreender os pontos de vista dos estudantes e suas experiências de interação com os artefatos de software. Durante a sessão do grupo focal, foi possível, com base nas avaliações dos participantes sobre o sistema de recomendação, responder as seguintes perguntas: "apresentava algum erro?", "estava de acordo com as características dos estudantes?", "o sistema foi capaz de se adaptar as mudanças de interesses dos estudantes?", "os conteúdos recomendados foram suficientes?" e "o sistema foi preciso quanto ao tempo de resposta?". Assim, os resultados apresentaram indícios de que tanto o funcionamento do SR, quanto a integração com a Youubi API foi considerada satisfatória. 
VII Congresso Brasileiro de Informática na Educação (CBIE 2018)

Anais do XXIX Simpósio Brasileiro de Informática na Educação (SBIE 2018)

Entretanto, de acordo com os resultados, pôde-se verificar que o componente apresentou limitações em relação à quantidade de conteúdos recomendados da Wikipédia; fato que ocorreu devido à quantidade de interações dos estudantes em conteúdos de vídeo ter sido bem maior, o que acabou levando ao SR priorizar esse tipo de conteúdo nas recomendações de forma desequilibrada. A abordagem do SR híbrido realizada neste trabalho pode ser melhorada, bem como verificar o quanto o SR está preciso por meio do uso de métricas.

\section{Referências}

BARJASTEH, I. et al (2015). Cold-start item and user recommendation with decoupled completion and transduction. In: Proceedings of the 9th ACM Conference on Recommender Systems. ACM, p. 91-98.

BARROS, P. A. (2016). Sistema de Filtragem Colaborativa: Um Estudo de Caso para Venda e Recomendação de Marmitas. Repositório de Relatórios-Sistemas de Informação, v. 1, n. 1.

BURKE, R. (2002). Hybrid recommender systems: Survey and experiments. User modeling and user-adapted interaction, v. 12, n. 4, p. 331-370.

EDUBI. (2018). Disponível: <http://edubi.ufersa.edu.br> Acesso: 13 de junho de 2018.

GARIN, R. S. et al (2006). O uso de técnicas de recomendação em um sistema para apoio à aprendizagem colaborativa. Revista Brasileira de Informática na Educação. Vol. 14, n. 3 (set./dez. 2006), p. 49-59.

GLUZ, J.; MACHADO, F.; GALÃO, M. (2017) Avaliação de Tecnologias de Bancos de Dados Semânticos para a Construção de um Sistema Inteligente de Gestão de Conteúdos de Aprendizagem: Experimentos e Resultados. Simpósio Brasileiro de Informática na Educação. p. 334.

ISOTANI, S.; BITTENCOURT, I. I. (2015). Dados Abertos Conectados: Em busca da Web do Conhecimento. Novatec Editora.

MEDIAWIKI. (2018). Welcome to MediaWiki.org. Disponível em: $<$ https://www.mediawiki.org/wiki/MediaWiki> Acesso em 13 de junho de 2018.

MONTEIRO, B. de S. (2015). Ambiente de aprendizado ubíquo Youubi: design e avaliação. Tese de doutorado em Ciência da Computação. UFPE, Recife, 2015.

REZENDE, P. A. A. et al. (2015) PERSONNA: proposta de ontologia de contexto e perfil de alunos para recomendação de objetos de aprendizagem. Revista Brasileira de Informática na Educação, v. 23, n. 01, p. 70.

TARUS, J. K.; NIU, Z.; YOUSIF, A. (2017). A hybrid knowledge-based recommender system for e-learning based on ontology and sequential pattern mining. Future Generation Computer Systems, v. 72, p. 37-48.

VIEIRA, F. J. R.; NUNES, M. A. S. N. (2012) Dica: Sistema de recomendação de objetos de aprendizagem baseado em conteúdo. Scientia Plena, v. 8, n. 5.

YOUTUBE-DEV (2018). Youtube API Reference, Disponível em: https://developers.google.com/youtube/v3/docs/ Acesso em: 27 de Abr de 2018. 